\title{
EXTREME POINTS OF CLASSES OF FUNCTIONS DEFINED BY SUBORDINATION
}

\author{
D. J. HALLENBECK ${ }^{1}$
}

ABSTRACT. The closed convex hull and extreme points of families of analytic functions, which are defined in terms of subordination, are determined. Integral representations are given for the hulls of the se families in terms of probability measures on suitable sets. These results are used to solve extremal problems. The functions we consider are defined by subordination to certain classes of starlike and convex mappings.

1. Introduction. In this paper, we shall determine the extreme points and the convex hulls of families of analytic functions, which are defined in terms of subordination. In [5], T. H. MacGregor and this author initiated this study. We shall extend some results of that paper and give some applications of new results.

Let $\Delta$ denote the unit disk. We say $f$ is subordinate to $F$, written $f \prec F$, if there exists an analytic function $\phi$, known as a Schwarz function, so that $\phi(0)=0,|\phi(z)|<1$ and $f(z)=F(\phi(z))$. We let St $(\alpha)$, where $\alpha<1$, denote the set of functions which are starlike of order $\alpha$. We recall that $f(z) \in \dot{\mathrm{St}}(\alpha)$ if and only if $f(0)=0, f^{\prime}(0)=1$ and $\operatorname{Re}\left(z f^{\prime}(z) / f(z)\right)>a$ for $z$ in $\Delta$. We let $K(\alpha)$, where $\alpha<1$, denote the set of functions which are convex of order $\alpha$. We recall that $f(z) \in K(\alpha)$ if and only if $f(0)=0, f^{\prime}(0)=1$, and $\operatorname{Re}\left(1+z f^{\prime \prime}(z) / f^{\prime}(z)\right)>\alpha$ for $z$ in $\Delta$. We note that $\operatorname{St}(\alpha)$ and $K(\alpha)$ are both compact families of analytic functions. We let $\mathrm{St}^{*}(\alpha)=\{f: f<g$ for some $g$ in St $(\alpha)\}$ and $K^{*}(\alpha)=\{f: f \prec g$ for some $g$ in $K(\alpha)\}$. In [6, p. 365], T. H. MacGregor proved that for any compact family $F$ of analytic functions $F^{*}=$ $\{f: f \prec g$ for some $g$ in $F\}$ is a compact family. Hence $\operatorname{St}^{*}(\alpha)$ and $K^{*}(\alpha)$ are compact. We let $S$ dinote the set of analytic functions on $\Delta$ which are univalent and satisfy $f(0)=0, f^{\prime}(0)=1$. It is known that $\operatorname{St}(\alpha) \subset S$ for $0 \leq \alpha<1$

Received by the editors July 26, 1973.

AMS (MOS) subject classifications (1970). Primary 30A32.

Key words and phrases. Starlike functions, convex functions, close-to-convex function, extreme point, probability measures, integral representation, subordination.

1 This research was, in part, supported by a University of Delaware Faculty Research Summer Grant. 
and $K(\alpha) \subset S$ for $-1 / 2 \leq \alpha<1$. For any compact family of analytic functions $F$ we let $\mathcal{H}_{F}$ denote the closed convex hull of $F$ and $\mathcal{E} \mathcal{H}_{F}$ denote the extreme points of the closed convex hull。

We shall determine $\mathcal{H S t}^{*}(\alpha)$ for $\alpha=1 / 2$ and $\alpha \leq 0$. We also determine $\mathcal{H} K^{*}(\alpha)$ for $\alpha \leq 0$.

2. Extreme points and convex hull of $K^{*}(\alpha)$.

Theorem 1. Let $X \times Y=\{(x, y):|x|=|y|=1\}, \mathcal{P}$ be the set of probability measures on $X \times Y, \alpha \leq 0$, and $\mathcal{F}$ be the set of functions $f_{\mu}$ on $\Delta$ defined by

$$
f_{\mu}(z)=\int_{X \times Y} \frac{1}{1-2 \alpha} \frac{x}{y}\left(\frac{1}{(1-y z)^{1-2 a}}-1\right) d \mu(x, y) \quad(\mu \in \mathcal{P}) ;
$$

then $\mathcal{H} K^{*}(\alpha)=\mathcal{F}$ and

$$
\mathscr{E H}\left(K^{*}(\alpha)=\left\{(1-2 \alpha)^{-1} x y^{-1}\left((1-y z)^{-(1-2 \alpha)}-1\right):|x|=|y|=1\right\} .\right.
$$

Proof. Suppose $f \in \mathcal{E} \mathfrak{H} K^{*}(\alpha)$; then, as was pointed out in [6, p. 366], $f<g$ for some function $g \in \mathcal{E H} K(\alpha)$. In Theorem 4 [2] the set $\mathscr{E H} K(\alpha)$ was proved to be the set of functions

$$
g(z)=(1-2 \alpha)^{-1} y^{-1}\left((1-y z)^{-(1-2 \alpha)}-1\right) \quad \text { for }|y|=1 .
$$

So

$$
f(z)=(1-2 \alpha)^{-1} y^{-1}\left((1-y \phi(z))^{-(1-2 \alpha)}-1\right)
$$

for some $y$ satisfying $|y|=1$ and some Schwarz function $\phi(z)$.

Let

$$
K(y)=\left\{f: f<g, g(z)=(1-2 \alpha)^{-1} y^{-1}\left((1-y z)^{-(1-2 \alpha)}-1\right)\right\} .
$$

Since $K(y) \subset K^{*}(\alpha)$ we conclude that $\mathcal{H} K(y) \subset \mathcal{H} K^{*}(\alpha)$ and $f \in \mathcal{E} H(K(y)$. Let $H=\left\{h: h \prec(1-z)^{-(1-2 a)}\right\}$ 。 The map $h \rightarrow(1-2 a)^{-1} y^{-1}(h(z)-1)$ is a linear map from $H$ to $K(y)$ and consequently from $\mathcal{E H} H$ to $\mathscr{E H} K(y)$. We recall that in Theorem 2.2 [1] $\mathcal{E} \mathcal{H}_{H}$ was determined to be the set $\left\{(1-x z)^{-(1-2 \alpha)}\right.$ : $|x|=1\}$ since $\alpha \leq 0$ implies that $1-2 \alpha \geq 1$. Hence we see that

$f(z)=(1-2 \alpha)^{-1} y^{-1}\left((1-x z)^{-(1-2 a)}-1\right)=(1-2 \alpha)^{-1} x(y x)^{-1}\left((1-x z)^{-(1-2 \alpha)}-1\right)$

which by a renaming of letters becomes

$$
(1-2 \alpha)^{-1} x y^{-1}\left((1-y z)^{-(1-2 \alpha)}-1\right) .
$$


Hence

$\mathscr{E} H\left(K^{*}(\alpha) \subset E=\left\{(1-2 \alpha)^{-1} x y^{-1}((1-y z)-(1-2 \alpha)-1):|x|=|y|=1\right\}\right.$.

We prove $E \subset \mathcal{E} H K^{*}(\alpha)$ by showing that each function in $E$ uniquely maximizes a real-valued continuous linear functional over $E$. We note that

$$
f(z)=(1-2 \alpha)^{-1} x y^{-1}\left((1-y z)^{-(1-2 \alpha)}-1\right)
$$

is in $K^{*}(\alpha)$ for each pair $x, y$ satisfying $|x|=|y|=1$. Let $J(f)=a f^{\prime}(0)+b f^{\prime \prime}(0)$ where $a=x^{-1}$ and $b=(x y)^{-1}$ for a fixed pair $|x|=|y|=1$. For any $f(z) \in E$ we see that $\operatorname{Re} J(f) \leq 3-2 \alpha$ and that $\operatorname{Re} J(f)=3-2 \alpha$ if and only if $x=a^{-1}$ and $x y=b^{-1}$. Hence, for the choice of $a$ and $b$ indicated above $\operatorname{Re} J(f)=3-2 \alpha$. So a unique func-

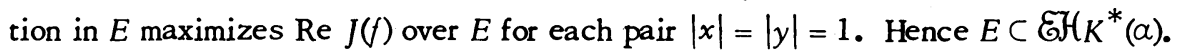

The conclusion of the theorem now follows from the Krein-Milman theorem $[4$, p. 440] and Theorem 1 in [3, p. 93].

Remarks. 1. This result generalizes Theorem 1 in [5] where $\mathcal{H} K^{*}$ is determined.

2. We recall that $K(\alpha) \subset \mathcal{C}$ when $-1 / 2 \leq \alpha<0$ and $\mathcal{C}$ denotes the class of close-to-convex functions. By applying the preceding theorem, we see that

$$
\mathscr{G}\left(K^{*}(-1 / 2)=\left\{z-(1 / 2) x z^{2}(1-x z)^{-2}:|x|=1\right\} .\right.
$$

We next introduce some notation. Suppose $f(z)=\sum_{n=0}^{\infty} a_{n} z^{n}$ and $F(z)=$ $\sum_{n=0}^{\infty} A_{n} z^{n}$ are analytic functions in $\Delta$. Suppose $\left|a_{n}\right| \leq\left|A_{n}\right|$ for $n=0,1,2$, $\cdots$. We denote this relation by $f \ll F$.

Theorem 2. If $f(z) \prec F(z), F(z) \in K(\alpha)$, and $\alpha \leq 0$, then

$$
f(z) \ll(1-2 \alpha)^{-1}\left((1-z)^{-(1-2 \alpha)}-1\right) .
$$

Proof. Since it suffices to consider as candidates for $F(z)$ the functions in $\mathscr{E H} K^{*}(\alpha)$, the result follows immediately from the previous theorem.

3. Extreme points and convex hull of $\mathrm{St}^{*}(a)$.

Theorem 3. Let $X \times Y$ and $\mathcal{P}$ be as in Theorem $1, \alpha=1 / 2$ or $\alpha \leq 0$, and $\mathcal{F}$ be the set of functions $f_{\mu}$ analytic on $\Delta$ defined by

$$
f_{\mu}(z)=\int_{X \times Y} \frac{y z}{(1-x z)^{2-2 a}} d \mu(x, y) \quad(\mu \in \mathcal{P}) .
$$

Then $\mathcal{H S t}^{*}(\alpha)=\mathcal{F}$ and $\mathcal{G H S t}^{*}(\alpha)=\left\{y z(1-x z)^{-(2-2 \alpha)}:|x|=|y|=1\right\}$.

Proof. Suppose $\alpha \leq 0$ and $f \in \mathcal{E} \mathcal{H S t}^{*}(\alpha)$, then as remarked earlier we have $f \prec g$ for some function $g$ in $\operatorname{GHSt}(\alpha)$. But in [2, Theorem 3] the set 
$\mathcal{E H} \operatorname{st}(\alpha)$ was determined to be the set of functions $\left\{g(z)=z(1-x z)^{-(2-2 a)}\right.$ : $|x|=1\}$ 。 Hence $f(z)=\phi(z)(1-x \phi(z))^{-(2-2 a)}$ for some $x$ satisfying $|x|=1$ and some Schwarz function $\phi(z)$.

Let $A=\left\{f: f \prec z(1-x z)^{-(2-2 a)},|x|=1\right\}$. It is clear that

$$
y z(1-x z)^{-(2-2 a)} \in A
$$

for each pair $|x|=|y|=1$, since $y z(1-x z)^{-(2-2 a)}=\phi(z)(1-x \bar{y} \phi(z))^{-(2-2 a)}$ where $\phi(z)=y z$. Hence $\mathcal{F} \subset \mathcal{H} A$. We next show that $A \subset \mathcal{F}$.

Suppose $f(z) \prec z(1-x z)^{-(2-2 a)}$ for some $|x|=1$. Hence

$$
f(z)=\frac{1}{(1-x \phi(z))^{1-2 \alpha}} \frac{\phi(z)}{1-x \phi(z)}=\int_{X} \frac{1}{(1-x z)^{1-2 \alpha}} d \mu(x) \int_{Y \times W} \frac{y z}{1-w z} d v(y, w)
$$

where $X=\{x:|x|=1\}, Y=\{y:|y|=1\}$ and $W=\{w:|w|=1\}$. This follows by Theorem 1 of [5] and Theorem 2.2 of [1] since $1-2 \alpha \geq 1$. Hence we have by the Fubini theorem

$$
f(z)=\int_{X \times(Y \times W)} \frac{y z}{(1-x z)^{1-2 a(1-w z)}} d \mu(x) d v(y, w) .
$$

It suffices to prove $y z(1-x z)^{-(1-2 \alpha)}(1-w z)^{-1} \in \mathcal{F}$ since $\mathcal{F}$ is closed and convex。However, by Theorem 1 of [2] we have

$$
\frac{y z}{(1-x z)^{1-2 a}(1-w z)}=\int_{T} \frac{y z}{(1-t z)^{2-2 a}} d n(t)
$$

where $T=\{t:|t|=1\}$ and $n(t)$ is a probability measure on $T$. Hence, it sufo fices to prove that $y z(1-t z)^{-(2-2 \alpha)}$ is in $\mathcal{F}$ for each pair $|y|=|t|=1$ 。 This is clearly true and hence $A \subset \mathcal{F}$ and so $\mathcal{H}_{A} \subset \mathcal{H F}=\mathcal{F}$.

As remarked above, we know that $\mathcal{E H S t}^{*}(\alpha) \subset A$. Hence $\mathcal{H S t}^{*}(\alpha)=$ $\mathcal{H E H S t}{ }^{*}(\alpha) \subset \mathcal{H} A=\mathcal{F}$ by the Krein-Milman theorem since $\mathcal{H S t}^{*}(\alpha)$ is compact and convex.

$$
\text { Since } y z(1-x z)^{-(2-2 a)}=\phi(z)(1-x \bar{y} \phi(z))^{-(2-2 a)} \text { where } \phi(z)=y z
$$
we conclude that $y z(1-x z)^{-(2-2 \alpha)}$ is in St $^{*}(\alpha)$ for each pair $|x|=|y|=1$ 。 Hence $\mathfrak{F} \subset \mathcal{H S t}^{*}(\alpha)$ by Theorem 1 [3] and consequently $\mathcal{H S t}^{*}(\alpha)=\mathcal{F}$.

Since $\mathcal{H S t}^{*}(\alpha)=\mathcal{F}$ we have $\mathcal{H H S t}^{*}(\alpha) \subset E$ where $E=\left\{y z(1-x z)^{-(2-2 a)}\right.$ : $|x|=|y|=1\}$ 。 To prove $E \mathcal{H S t}^{*}(\alpha)=E$ we need only show that each function in $E$ uniquely maximizes a real-valued continuous linear functional over $E$ 。 This follows as earlier in part because $E \subset \mathrm{St}^{*}(\alpha)$.

Let $J(g)=u g^{\prime}(0)+v g^{\prime \prime}(0)$ where $|u|=|v|=1$. We then have

$\operatorname{Re} J(g)=\operatorname{Re}\{u y+2(2-2 \alpha) v y z\} \leq|u v|+4(1-\alpha)|v y z|=5-4 \alpha$. 
The maximum value of $5-4 \alpha$ is only achieved if $y=u^{-1}$ and $x=u / v$. Letting $u$ and $v$ vary, we get all possible pairs $x, y$ associated with the unique function in $E$ with $\operatorname{Re} J(g)=5-4 a$. Hence $E \subset \mathcal{E H S t}^{*}(\alpha)$ and consequently $\mathscr{E} H_{S t}{ }^{*}(\alpha)=E$.

Remarks. 1. Theorem 3 generalizes Theorem 2 in [5] by the present author and T.H. MacGregor.

2. We recall that Theorem 3 in [2] when $\alpha=1 / 2$ and $k=1$ showed that $\mathcal{G H S t}(1 / 2)=\mathcal{G H} K$ where the latter set was determined in Theorem 2 in $[3, \mathrm{p}$. 94]. This fact, coupled with an examination of the proof in Theorem 1 of this paper when $\alpha=0$, shows that Theorem 3 above holds when $\alpha=1 / 2$.

3. It was proven in Theorem 7 in [2] that if $f<F$ where $F$ is in $\operatorname{St}(\alpha)$ and $1 / 2 \leq \alpha<1$ then $f(z)<<z /(1-z)$. Hence Theorem 3 cannot hold when $1 / 2<\alpha<1$. So $\mathcal{H S t}{ }^{*}(\alpha) \not \subset \mathcal{F}$ when $1 / 2<\alpha<1$. We conjecture that $\mathrm{HSt}{ }^{*}(\alpha)=\mathfrak{F}$ when $0<a<1 / 2$ but we have been unable to prove this.

4. Theorem 3 affords a quick proof of Theorem 7 in [2]. If $f \prec F$ and $F \in \operatorname{St}(\alpha)$ when $\alpha \leq 0$ then $F(z)<<z(1-z)^{-(2-2 \alpha)}$ and if $\alpha=1 / 2$ then $f(z)<</(1-z)$.

4. A coefficient estimate for functions in $\mathrm{St}^{*}(\alpha)$ when $0<\alpha<1 / 2$.

Theorem 4. If $f(z)=\sum_{n=1}^{\infty} a_{n} z^{n} \prec F(z)$ where $F(z) \in \operatorname{St}(\alpha)$ and $0<\alpha<1 / 2$ then $\left|a_{n}\right|<(1-2 \alpha) n+2 \alpha=B(\alpha)$.

Proof. It suffices to consider $F(z)=z /(1-z)^{2-2 \alpha}$. We have

$$
f(z)=\frac{\phi(z)}{1-\phi(z)} \frac{1}{(1-\phi(z))^{1-2 \alpha}} .
$$

We let $\phi(z) /(1-\phi(z))=b_{1} z+b_{2} z^{2}+\cdots$ and $(1-\phi(z))^{-(1-2 a)}=1+c_{1} z+$ $c_{2} z^{2}+\cdots$. We have $a_{n}=b_{n}+b_{n-1} c_{1}+b_{n-2} c_{2}+\cdots+b_{1} c_{n-1}$ and we know that $\left|b_{n}\right| \leq 1$ for $n=1,2, \cdots$ by Theorem 1 when $\alpha=0$ or by the classical result of Rogosinski [7]. We also know that $\left|c_{n}\right| \leq 1-2 a$ for $n=1,2, \cdots$ by another theorem of Rogosinski [7] with equality never hold. ing for two values of $n$ simultaneously. Hence by the triangle inequality $\left|a_{n}\right| \leq(1-2 a) n+2 \alpha$. Equality is not possible since $\left|c_{n}\right|$ cannot equal $1-2 \alpha$ for more than a single value of $n$.

Remarks. We note that $B(0)=n$, the classical result of Rogosinski [7] and $B(1 / 2)=1$ which is contained in Theorem 7 in [2]. We conjecture that $f(z)<<z /(1-z)^{2-2 a}$ which is known to hold for $\alpha \leq 0$ also holds for $0<a<1 / 2$. 


\section{REFERENCES}

1. D. A. Brannan, J. G. Clunie and W. E. Kirwan, On the coefficient problem for functions of bounded boundary rotation, Ann. Acad. Sci. Fenn. AI 523 (1973).

2. L. Brickman, D. J. Hallen beck, T. H. MacGregor and D. R. Wilken, Convex hulls and extreme points of families of starlike and convex mappings, Trans. Amer. Math. Soc. 185 (1972), 413-428.

3. L. Brickman, T. H. MacGregor and D. R. Wilken, Convex hulls of some classical families of univalent functions, Trans. Amer. Math. Soc. 156 (1971), 91107. MR. 43 \#494.

4. N. Dunford and J. T. Schwartz, Linear operators. I: General theory, Pure and Appl. Math., vol. 7, Interscience, New York, 1958. MR 22 \#8302.

5. D. J. Hallenbeck and T. H. MacGregor, Subordination and extreme point theory, Pacific J. Math. (to appear).

6. T. H. MacGregor, Applications of extreme point theory to univalent functions, Michigan Math. J. 19 (1972), 361-376.

7. W. Rogosinski, On the coefficients of subordinate functions, Proc. London Math. Soc. (2) 48 (1943), 48-82. MR 5, 36.

DE PARTMENT OF MATHEMATICS, UNIVERSITY OF DELAWARE, NEWARK, DELAWARE 19711 